\title{
Validity and reliability of Persian version of quality of life questionnaire in people living with HIV/AIDS (WHOQOL-HIV-BREF)
}

\author{
Azizallah Dehghan ${ }^{1,2}$, Zeinab Heidari $^{3}$, Jalal Karimi ${ }^{3}$, Aboubakr Jafarnezhad ${ }^{4,5}$, Sanaz Atef ${ }^{1}$, Hamid Sharifi², \\ Mojtaba Farjam ${ }^{1}$ \\ ${ }^{1}$ Noncommunicable Diseases Research Center, Fasa University of Medical sciences, Fasa, Iran \\ ${ }^{2}$ HIV/STI Surveillance Research Center, and WHO Collaborating Center for HIV Surveillance, Institute for Futures Studies \\ in Health, Kerman University of Medical, Kerman, Iran \\ ${ }^{3}$ Fasa University of Medical Sciences, Fasa, Iran \\ ${ }^{4}$ Hormozgan University of Medical Sciences, Bandar Abbas, Iran \\ ${ }^{5}$ Neurology Research Center, Kerman University of Medical Sciences, Kerman, Iran
}

\begin{abstract}
Introduction: The aim of this study was to assess validity and reliability of Persian version of quality of life (QOL) questionnaire in people living with human immunodeficiency virus (HIV)/acquired immunodeficiency syndrome (AIDS) (WHOQOL-HIV-BREF).

Material and methods: A total of 150 HIV/AIDS patients who were referred to Fasa and Larestan Behavioral Disease Counseling Centers were recruited into the study. WHOQOL-HIV-BREF questionnaire was completed by trained interviewers. Reliability of questionnaire was measured using Cronbach's a coefficient. Construct validity was assessed by item-scale correlation method, and criterion validity was determined with discriminant validity and convergent validity.

Results: Cronbach's a was computed above 0.8 for all dimensions of the questionnaire. Item-convergent validity ranged from 0.38 to 0.83 . QOL score was higher in AIDS patients compared with HIV-infected individuals in all dimensions $(p<0.01)$.

Conclusions: The findings of this study show that WHOQOL-HIV-BREF questionnaire provide acceptable validity and reliability for measuring QOL among Persian-speaking HIV/AIDS-infected patients in Iran.
\end{abstract}

HIV AIDS Rev 2021; 20, 4: 270-274

DOI: https://doi.org/10.5114/hivar.2021.111877

Key words: HIV, AIDS, validity, reliability, Persiane.

\section{Introduction}

Human immunodeficiency virus (HIV) and acquired immunodeficiency syndrome (AIDS) are chronic and debilitating illnesses, imposing a significant burden on patient quality of life (QOL) and causing physical, mental, and finan- cial harms [1]. According to the World Health Organization, globally, about 36.9 million people were living with HIV in 2017 (95\% CI: 31.1-43.9) [2], and Iran is one of the countries with the most noteworthy HIV/AIDS rate in the Middle East [3]. Moreover, HIV/AIDS is estimated to be account-
Address for correspondence: Prof. Azizallah Dehghan, Noncommunicable Diseases Research Center, Fasa University of Medical Sciences, Fasa, Iran, e-mail: dehghan.aziz@yahoo.com
Article history:

Received: 25.10 .2020

Received in revised form: 18.03.2021

Accepted: 25.03.2021

Available online: 30.11 .2021
International Journal of HIV-Related Problem

HIV \& AIDS

$R$ e vi e w 
ed for the highest burden of disease in Iran by the year 2025 [4]. These patients often experience social exclusion and discrimination in employment, housing, insurance, medical care, etc., resulting in poor QOL as well as bad physical and mental health [5].

Life expectancy is usually considered in the treatment of HIV/AIDS patients, while patients' QOL should also be taken into account [6]. Given recent advances in the treatment of HIV/AIDS and subsequently elevated survival rate of these patients, their QOL is increasingly becoming more important, and has drawn the attention of health policymakers and healthcare providers [7].

The World Health Organization has defined quality of life as "a person's perception of its' own position in life in the context of culture and value systems, in which they live and in association with their expectations, goals, standards, and concerns" [8-10].

Measurement of QOL is usually performed using general or disease-specific questionnaires. Earlier tool is used to measure QOL in a wide range of patients (e.g., cardiovascular, pulmonary, cancer, AIDS, etc.) and healthy people, whereas later instrument is effective in assessment of healthrelated QOL (HRQOL) for a specific disease [11].

WHOQOL-HIV-BREF is a proprietary and short-range method for assessing QOL of HIV/AIDS patients, which can be used to evaluate QOL of individuals with different cultural backgrounds. This questionnaire was first developed by the World Health Organization and recommended for measuring QOL in HIV/AIDS patients $[12,13]$. In order to use an instrument originated from a different language and culture, appropriate validity and reliability must be adopted [14]. Therefore, this study was conducted to assess the validity and reliability of Persian version of QOL questionnaire - WHOQOL-HIV-BREF.

\section{Material and methods}

This was a cross-sectional study. In order to appropriately employ a questionnaire that originate from different country and language, the following steps should be followed: 1) translate and back translate the questionnaire, and match with an original version; 2) cultural adaptation of questions, and a correction if the question is unclear; 3) validity and reliability of the questionnaire must be measured [6].

\section{Data collection instrument}

WHOQOL-HIV-BREF is a 31 -item instrument, in which 26 questions are similar to those of WHOQOL-BREF questionnaire and 5 items are devoted to AIDS/HIV. This questionnaire is divided into six broad domains of quality of life, including physical domain, psychological domain, levels of independence, social relationships, environment, and spiritual domain. The first two questions of the scale are unrelated to QOL domains and assess overall attitude of individuals towards QOL and health status [15].
Each item of WHOQOL-HIV-BREF is rated on a 5-point Likert scale, scored from 1 to 5 in most questions, with score 1 meaning 'very poor impression' and score 5 denoted as 'very good positive impression'. In questions where a higher score does not mean a better quality of life, when calculating their scores, the scores of the answers are reversed. To calculate the score of each domain, the score of the questions in each domain is added together and divided by the number of questions in that domain, and then multiplied by 4 . Each dimension score ranges from 4 to 20 , where 4 is the worst and 20 is the best position in that dimension [15].

It should be noted that the Persian version of WHOQOL-BREF questionnaire in Iran, provides acceptable validity and reliability [16]. However, in this study, the common questions between questionnaires 1 and 2 were translated independently, and translations are very similar. English version of the questionnaire was translated into Persian by an epidemiologist and 2 English language specialists. Then, a single Persian version was prepared. In the next step, the Persian version was translated into English by 3 English language specialists, and out of these three versions, a final English version was prepared and compared with the original English version of the questionnaire.

\section{Study population}

According to Fayer et al., a sample size of 100 to 400 individuals was considered appropriate for assessing reliability

Table 1. Demographic and clinical features of the participants

\begin{tabular}{l|c}
\hline Variables & $n(\%)$ \\
\hline $\begin{array}{l}\text { Gender } \\
\text { Male }\end{array}$ & $84(65.1)$ \\
\hline Female & $45(34.9)$ \\
\hline \begin{tabular}{l} 
Marital status \\
\hline Single
\end{tabular} \\
\hline Married & $66(51.2)$ \\
\hline Widow & $48(37.2)$ \\
\hline Divorced & $6(4.7)$ \\
\hline Education level & $9(7.0)$ \\
\hline Under diploma & $69(53.5)$ \\
\hline Diploma & $54(41.9)$ \\
\hline Academic & $6(4.7)$ \\
\hline Place of living & \\
\hline Urban & $81(62.8)$ \\
\hline Rural & $48(37.2)$ \\
\hline Stage of disease & $93(72.1)$ \\
\hline HIV infection & $36(27.9)$ \\
\hline AlDS & $129(100)$ \\
\hline Total & \\
\hline
\end{tabular}


Table 2. Result of factor analysis by varimax rotation

\begin{tabular}{|c|c|c|c|c|c|c|c|}
\hline \multicolumn{2}{|c|}{ Question } & \multicolumn{6}{|c|}{ Component } \\
\hline & & \multirow{2}{*}{$\begin{array}{c}1 \\
0.677 \\
\end{array}$} & \multirow{2}{*}{$\begin{array}{c}2 \\
-0.321\end{array}$} & \multirow{2}{*}{$\begin{array}{c}3 \\
-0.147\end{array}$} & \multirow{2}{*}{$\begin{array}{c}4 \\
-0.097\end{array}$} & \multirow{2}{*}{$\begin{array}{c}5 \\
0.045\end{array}$} & \multirow{2}{*}{$\begin{array}{c}6 \\
-0.047 \\
\end{array}$} \\
\hline 1 & Pain and discomfort & & & & & & \\
\hline 2 & Bothered by any physical problems related to HIV & 0.726 & -0.067 & -0.236 & 0.122 & -0.126 & 0.130 \\
\hline 3 & Dependence on medication or treatment & -0.232 & -0.047 & 0.600 & 0.216 & 0.077 & -0.368 \\
\hline 4 & Positive feeling & 0.419 & 0.504 & -0.200 & -0.384 & -0.066 & 0.206 \\
\hline 5 & Religion, spirituality, and personal beliefs & 0.335 & 0.038 & -0.118 & -0.207 & 0.503 & -0.013 \\
\hline 6 & Forgiveness and blame & -0.129 & -0.038 & -0.139 & 0.047 & 0.635 & 0.059 \\
\hline 7 & Concerns about the future & -0.056 & -0.125 & 0.428 & -0.133 & 0.628 & -0.221 \\
\hline 8 & Death and dying & -0.260 & -0.007 & 0.019 & -0.178 & 0.678 & 0.067 \\
\hline 9 & Thinking, learning, memory, and concentration & 0.336 & 0.674 & -0.053 & -0.049 & 0.125 & -0.130 \\
\hline 10 & Physical safety and security & 0.291 & 0.330 & 0.072 & -0.445 & 0.051 & 0.462 \\
\hline 11 & Physical environment & 0.153 & 0.380 & -0.047 & 0.289 & 0.133 & 0.653 \\
\hline 12 & Energy and fatigue & 0.684 & 0.112 & -0.378 & -0.245 & 0.163 & 0.027 \\
\hline 13 & Bodily image and appearance & 0.225 & 0.486 & 0.336 & -0.187 & 0.035 & -0.221 \\
\hline 14 & Financial resources & 0.218 & 0.107 & 0.147 & -0.195 & 0.247 & 0.774 \\
\hline 15 & Social inclusion & 0.289 & -0.087 & -0.026 & 0.714 & 0.141 & 0.082 \\
\hline 16 & New information and skills & -0.270 & 0.199 & -0.082 & 0.225 & -0.025 & 0.626 \\
\hline 17 & Leisure activities & 0.275 & 0.201 & -0.046 & 0.254 & -0.102 & 0.650 \\
\hline 18 & Activities of daily living & -0.236 & 0.271 & 0.667 & 0.047 & -0.191 & -0.027 \\
\hline 19 & Sleep and rest & 0.509 & 0.097 & -0.136 & 0.237 & 0.280 & 0.489 \\
\hline 20 & Work capacity & -0.319 & 0.210 & 0.685 & 0.061 & -0.001 & 0.156 \\
\hline 21 & Mobility & -0.053 & -0.007 & 0.723 & -0.212 & 0.082 & 0.317 \\
\hline 22 & Self-esteem & 0.284 & 0.621 & -0.102 & -0.443 & 0.148 & 0.074 \\
\hline 23 & Personal relationships & -0.173 & 0.100 & -0.015 & 0.824 & 0.222 & -0.008 \\
\hline 24 & Sexual activity & -0.122 & 0.102 & -0.228 & 0.672 & 0.280 & -0.136 \\
\hline 25 & Social support & 0.017 & 0.234 & -0.174 & 0.673 & 0.192 & 0.345 \\
\hline 26 & Home environment & 0.062 & -0.008 & -0.145 & -0.037 & 0.122 & 0.786 \\
\hline 27 & Health and social care: accessibility and quality & 0.080 & 0.109 & 0.015 & -0.115 & -0.261 & 0.629 \\
\hline 28 & Transport & 0.020 & -0.061 & -0.240 & 0.306 & 0.100 & 0.506 \\
\hline 29 & Negative feelings & -0.172 & 0.795 & 0.012 & 0.040 & 0.044 & -0.019 \\
\hline
\end{tabular}

1 - physical health; 2-psychological health; 3-level of independence; 4-social relationships; 5 -spiritual/religion/personal beliefs; 6 - environmental health

and validity of QOL questionnaires [17]. We used convenience method, and $129 \mathrm{HIV} / \mathrm{AIDS}$ patients were selected from Fasa and Larestan Behavioral Disease Counseling Center. In each of the two centers, a trained psychologist collected the required data. In this study, inclusion criteria were at least 18 years of age and lack of chronic concomitant disease.

\section{Validity, reliability, and statistical analysis}

Expert opinions were used to confirm face validity and content validity, with two epidemiologists, two infectious disease specialists, a nursing specialist, and a health edu- cation specialist, who confirmed face and content validity of the questionnaire.

Factor analysis was used to identify all dimensions of the questionnaire and compare it with the original version of WHOQOL-HIV-BREF. Factor analysis compared our Persian-language questionnaire with the original version, and determined whether identified dimensions in the Persian language version of WHOQOL-HIV-BREF match the original version [6]. Construct validity was assessed using correlation of the scores on each item with total scores of relevant dimension (item - convergent validity), and other dimensions (item - discriminant validity). In this method, correlation of each item with its' own dimension score should be above 0.4 and less than 0.4 with other domains. 
Table 3. Item scaling tests: Convergent and discriminant validity for WHOQOL-HIV dimensions

\begin{tabular}{l|c|c|c|c|c}
\hline Domains & $\begin{array}{c}\text { No. } \\
\text { of items }\end{array}$ & $\begin{array}{c}\text { Item-convergent validity } \\
\text { (range of correlation) }\end{array}$ & $\begin{array}{c}\text { Scaling } \\
\text { success }\end{array}$ & $\begin{array}{c}\text { Item-discriminant validity } \\
\text { (range of correlation) }\end{array}$ & $\begin{array}{c}\text { Internal consistency } \\
\text { (Cronbach's } \alpha \text { ) }\end{array}$ \\
\hline Physical & 4 & $0.46-0.84$ & $4 / 4(100 \%)$ & $0.24-0.39$ & 0.83 \\
\hline Psychological & 5 & $0.43-0.82$ & $5 / 5(100 \%)$ & $0.19-0.48$ & 0.78 \\
\hline Level of independence & 4 & $0.49-0.78$ & $4 / 4(100 \%)$ & $0.16-0.54$ & 0.82 \\
\hline Social relationship & 4 & $0.56-0.88$ & $4 / 4(100 \%)$ & $0.27-0.49$ & 0.79 \\
\hline Environment & 8 & $0.62-0.85$ & $8 / 8(100 \%)$ & $0.21-0.46$ & 0.81 \\
\hline Spirituality & 4 & $0.47-0.76$ & $4 / 4(100 \%)$ & $0.32-0.54$ & 0.72 \\
\hline
\end{tabular}

Table 4. Comparison of QOL score in all domains between participants with and without symptoms by Persian version of WHOQOL-HIV questionnaire

\begin{tabular}{l|c|c|c}
\hline \multirow{2}{*}{ Domain } & \multicolumn{2}{|c|}{ Stage of disease } & \multirow{2}{*}{$p$-value* } \\
\cline { 2 - 4 } & HIV $(n=93)$ & AIDS $(n=36)$ & \\
\hline Physical & $11.46 \pm 2.18$ & $9.63 \pm 2.30$ & $<0.001$ \\
\hline Psychological & $12.63 \pm 2.66$ & $10.60 \pm 2.29$ & 0.002 \\
\hline Level of independence & $12.48 \pm 2.50$ & $10.86 \pm 2.55$ & $<0.001$ \\
\hline Social relationship & $13.07 \pm 3.47$ & $10.08 \pm 4.00$ & $<0.001$ \\
\hline Environment & $12.82 \pm 2.25$ & $10.38 \pm 2.44$ & $<0.001$ \\
\hline Spirituality & $12.01 \pm 2.80$ & $10.66 \pm 3.32$ & 0.035 \\
\hline Total & $73.14 \pm 9.77$ & $65.57 \pm 9.26$ & $<0.001$ \\
\hline
\end{tabular}

*Mann-Whitney U-test

Clinical validity was also assessed through comparing the score of all dimensions of WHOQOL-HIV-BREF between HIV-positive subjects and AIDS patients using MannWhitney $U$-test.

In addition, to assess validity of the questionnaire in terms of internal consistency, Cronbach's a coefficient of all dimensions of the questionnaire was calculated.

\section{Results}

A total of 129 PLWHA were recruited into this study, of whom $84(65.1 \%)$ patients were males. The average age of the patients was $30.01 \pm 8.96$ years, ranging from 22 to 56 years. The mean duration of infection was $5.64 \pm 4.17$ years. In addition, minimum duration of HIV infection ranged from 1 to 19 years. Other characteristics of the participants are presented in Table 1.

Table 2 shows the results of factor analysis based on varimax and oblimin rotations. Accordingly, all identified dimensions in the Persian version of WHOQOL-HIV-BREF were similar to the original version. Furthermore, all items displayed factor loadings above 0.4 in their dimensions. However, 5 items (item No. 6, 9, 12, 21, and 24) had a factor loadings above 0.4 in their own dimension as well as other irrelevant dimensions, with a lower factor loadings in irrelevant dimensions.
Item-convergent and item-discriminant validity were calculated for inter-item and item-domain correlations using Spearman's correlation coefficient test.

The item-convergence validity was $100 \%$ in all dimensions (Table 3). All items of the questionnaire had a correlation coefficient higher than 0.4 with their own dimension $(p<0.05)$. The score for item-domain correlation was lower in irrelevant dimensions than in relevant dimension (item - discriminant validity). These results reflected acceptable construct validity of the Persian-language WHOQOL-HIVBREF.

Cronbach's $\alpha$ was computed higher than 0.8 for all dimensions, indicating adequate measure of internal consistency and acceptable reliability of the questionnaire. The total QOL scores and scores of all QOL dimensions were higher in PLWHA than in HIV-infected individuals, demonstrating clinical validity of the questionnaire, as shown in Table 4 .

\section{Discussion}

Factor analysis revealed that all identified dimensions for the Persian version of WHOQOL-HIV questionnaire were similar to the original WHOQOL-HIV-BREF (Table 2). However, questions No. 6, 9, 12, 21, and 24 displayed a factor loading greater than 0.4 in two dimensions, while factor loading for the item was higher in its' dimension compared with other dimensions. 
Item convergent validity was above 0.4 for all items, and scores for discriminant validity of all items were lower in their own domain, indicating acceptable construct validity of items of the questionnaire.

The findings of this study suggest that all dimensions of the questionnaire displayed a very good internal consistency and reliability. Cronbach's $\alpha$ was higher than 0.7 in all dimensions, which was a recommended and acceptable value. These results were also observed in Malaysian, Vietnamese, Brazilian, and Thai versions of WHOQOL-HIV-BREF. The lowest Cronbach's $\alpha$ coefficient was obtained in the spiritual dimension, which could be attributed to limited number of items and/or item contents of this dimension. Moreover, a lower reliability of this dimension has been reported in other studies.

According to the results of the present study, WHOQOLHIV-BREF demonstrated moderate to high item-domain correlations (Table 3). These results are supported by Saddki et al. in Malaysia [18] and Neemon et al. in Taiwan [19].

In this study, the clinical validity of the WHOQOL-HIV questionnaire was measured. The QOL scores in all dimensions and the overall score of WHOQOL-HIV questionnaire were less among PLWHA than in HIV-positive individuals, showing a good validity of the questionnaire and an indicator of severity of the disease.

This study was conducted in two cities located in Fars province, which is a small proportion of the population of Iran. However, their culture is to a large extent similar to Persian speakers in Iran, and this questionnaire can most likely be used in other Iranian Persian speakers. Therefore, it is clear that in non-Persian speakers of Iran, the use of this questionnaire is not recommended, even though they can also speak Persian.

The use of convenience sampling was the only limitation of the present study. This can reduce external validity, because only those HIV/AIDS patients who received treatment and counseling were enrolled into the study, therefore the results cannot be generalized to all HIV/AIDS patients. It should be noted that there are some patients who are not recognized and not under treatment.

\section{Conclusions}

The results of this study showed that the Persian version of WHOQOL-HIV-BREF provide the sufficient validity and reliability for assessing QOL in Persian-speaking HIV/AIDS patients in Iran.

\section{Conflict of interest}

The authors declare no conflict of interest.

\section{References}

1. Rai Y, Dutta T, Gulati AK. Quality of life of HIV-infected people across different stages of infection. J Happiness Studies 2010; 11: 61-69.

2. UNAIDS. Global HIV \& AIDS statistics -2018 fact sheet. Availableat: http://www.unaids.org/sites/default/files/media_asset/UNAIDS_ FactSheet_en.pdf.
3. National ACS. Islamic Republic of Iran AIDS Progress Report. Ministry of Health and Medical Education Tehran; 2015, p. 73.

4. Khajehkazemi R, Sadeghirad B, Karamouzian M, et al. The projection of burden of disease in Islamic Republic of Iran to 2025. PLoS One 2013; 8: e76881.

5. Nikoo Seresht Z, Rimaz S, Nedjat S, Motevallian SA, Saeepour N. The relationship between quality of life and social capital amongst people living with HIV/AIDS attending the Imam Khomeini Hospital Consultation Center for clients with risky behaviors in Tehran - 2011. Journal of School of Public Health and Institute of Public Health Research 2014; 11: 17-28.

6. Ghaem H, Borhani Haghighi A, Zeighami B, Dehghan A. Validity and reliability of the Persian version of the Parkinson disease quality of life (PDQL) questionnaire. Journal of Kerman University of Medical Sciences 2010.

7. Kohli RM, Sane S, Kumar K, Paranjape RS, Mehendale SM. Assessment of quality of life among HIV-infected persons in Pune, India. Qual Life Res 2005; 14: 1641-1647.

8. Bijani M, Jafarnejad A, Bazrafshan MR, Atef S, Sarvestani PS, Dehghan A. Comparison the health related quality of life in type 2 diabetic patients and healthy people: a cross-sectional study in Iran. Clin Diabetol 2021; 10: 370-374.

9. Gholami A, Jahromi LM, Zarei E, Dehghan A. Application of WHOQOL-BREF in measuring quality of life in health-care staff. Int J Prev Med 2013; 4: 809-817.

10. Jahanfar A, Fereidouni Z, Behnammoghadam M, Dehghan A, Bashti S. Efficacy of eye movement desensitization and reprocessing on the quality of life in patients with major depressive disorder: a randomized clinical trial. Psychol Res Behav Manag 2020; 13: 11-17.

11. Nejat S. Quality of life and its measurement. Iranian Journal of Epidemiology 2008; 4: 57-62.

12. Group W-H. Preliminary development of the World Health Organsiation's Quality of Life HIV instrument (WHOQOL-HIV): analysis of the pilot version. Social Science \& Medicine 2003; 57: 1259-1275.

13. Odili VU, Ikhurionan IB, Usifoh SF, Oparah AC. Determinants of quality of life in HIV/AIDS patients. West African Journal of Pharmacy 2011; 22: 42-48.

14. Dehghan A, Nasirian M, Haghdoost AA, Bahramali E, Sharifi H. Validation of the verbal autopsy questionnaire for adult deaths in Iran. Medical Journal of The Islamic Republic of Iran (MJRI) 2018; 32: 33-36.

15. Basavaraj KH, Navya MA, Rashmi R. Quality of life in HIV/AIDS. Indian J Sex Transm Dis AIDS 2010; 31: 75-80.

16. Nejat S, Montazeri A, Holakouie Naieni K, Mohammad K, Majdzadeh SR. The World Health Organization quality of Life (WHOQOLBREF) questionnaire: translation and validation study of the Iranian version. Journal of School of Public Health and Institute of Public Health Research 2006; 4: 1-12.

17. Fayers PM, Machin D. Quality of life: the assessment, analysis and interpretation of patient-reported outcomes. John Wiley \& Sons; 2013.

18. Saddki N, Noor MM, Norbanee TH, Rusli MA, Norzila Z, Zaharah $S$, et al. Validity and reliability of the Malay version of WHOQOL-HIV BREF in patients with HIV infection. AIDS Care 2009; 21: 1271-1278.

19. Meemon N, Paek SC, Yenchai D, Wan TTH. Application of the WHOQOL-HIV-BREF questionnaire in HIV-infected Thai patients: reliability and validity of the instrument. J Assoc Nurses AIDS Care 2016; 27: 698-708. 